\title{
Possibilidades do Pensamento Computacional: um novo olhar teórico
}

\author{
Carlos Roberto Beleti Junior $^{1}$, Marta Sueli de Faria Sforni ${ }^{2}$ \\ ${ }^{1}$ Universidade Federal do Paraná (UFPR) \\ Campus Jandaia do Sul - PR - Brasil \\ ${ }^{2}$ Universidade Estadual de Maringá (UEM) \\ Maringá - PR - Brasil \\ carlosbeleti@ufpr.br, martasforni@uol.com.br
}

\begin{abstract}
The emergence of new themes, when related to computing, can cause changes in the most diverse areas. In Education, a recent concept, Computational Thinking (CT), has moved the scene. Several researches have investigated the theme, its definition and structure, ways to carry out its development and evaluation. However, there is no consensus on the best way to promote its development. To collaborate with the area, we propose a research that investigates the development of CT under a new theoretical perspective, based on the assumptions of the Historical-Cultural Theory and Developmental Teaching Theory with a conceptual basis in the Computer Science.
\end{abstract}

Resumo. $O$ surgimento de novas temáticas, quando relacionadas à Computação, pode provocar mudanças nas mais diversas áreas. Na Educação, um conceito recente, o Pensamento Computacional (PC), tem movimentado o cenário. Diversas pesquisas têm investigado a temática, sua definição e estrutura, formas de realizar seu desenvolvimento e avaliação. No entanto, não há consenso sobre a melhor forma de promover seu desenvolvimento. Visando colaborar com a área, propomos uma pesquisa que investiga o desenvolvimento do PC sob uma nova perspectiva teórica, alicerçado sobre os pressupostos da Teoria Histórico-Cultural e da Teoria do Ensino Desenvolvimental, com base conceitual nos fundamentos da Ciência da Computação.

\section{Introdução}

O Pensamento Computacional (PC) tem sido amplamente discutido e abordado nos diversos níveis de ensino e nos mais variados campos de conhecimento, sobretudo na Educação e Computação. Após a publicação do artigo sobre o PC, de Jeannette Wing [Wing 2006], a quantidade de trabalhos primários, secundários e terciários sobre o PC tem aumentado significativamente por tudo o mundo, seja em eventos ou conferências e periódicos dessas áreas [Kaminski et al. 2021].

Apesar de pesquisas evidenciarem um histórico de precursores sobre a temática do PC, que se inicia em meados dos anos 40, com George Polya, mais a frente com Alan Perlis e Herb Simon na década de 60, e que continua nos anos subsequentes com Donald Knuth e Edsger Dijkstra, até chegar a Seymour Papert, em 1980 [Denning 2017], não há consenso quanto a uma definição global e sobre os fundamentos do PC.

Papert foi o primeiro a cunhar o termo $\mathrm{PC}$ fazendo menção à possibilidade de “integrar o pensamento computacional à vida cotidiana" [Papert 1980], no entanto, há 
apenas essa menção sobre o termo em sua obra. Décadas depois, em 2006, Wing reavivou a temática e, desde então, tem sido considerada a entusiasta do termo na chamada "nova versão do PC", em contraposição ao "PC tradicional" que, segundo [Denning 2017], relaciona o $\mathrm{PC}$, obrigatoriamente, ao uso de modelos computacionais. Temos, portanto, um histórico que traça as origens do $\mathrm{PC}$, formulado majoritariamente por pesquisadores da Ciência da Computação (CC), destacando principalmente aspectos computacionais, sobretudo programação, à exceção de Papert que, apesar de Matemático, desenvolveu sua base teórica nos preceitos de Jean Piaget junto ao processo de aprendizagem mediado pelo computador, também com foco na programação [Papert 1980].

Com exceção dessa aproximação com o campo da psicologia realizada por Papert, não houve significativas considerações sobre, por exemplo, a formação do pensamento ou mesmo do psiquismo em pesquisas que investigaram o PC. Todavia, se o PC envolve o processo do pensamento humano junto aos fundamentos da Computação [Wing 2008], não seria essencial um aporte teórico relacionado às bases do psiquismo, mais especificamente sobre uma das funções psicológicas superiores, o pensamento?

Esse questionamento geral nos orienta a investigar as bases teóricas da formação do pensamento humano visando compreender as formas de pensamento existentes, tais como pensamento teórico, pensamento lógico, pensamento matemático, entre outros, para, então, compreender a particularidade do PC. Trata-se do movimento de investigar sua gênese em um aporte teórico que tem como objeto de estudo o pensamento humano de modo geral e, então, retomar a discussão dessa área de pesquisa.

Desse modo, apresentamos as ações iniciais desta pesquisa, que tem o objetivo de investigar a essência do PC, tendo como bases conceituais os fundamentos da CC, da Teoria Histórico-Cultural (THC) e da Teoria do Ensino Desenvolvimental (TED). O trabalho em tela, buscará oferecer subsídios para responder a dois questionamentos que tem acompanhado a investigação de pesquisas na área:

- Além da CC, quais bases teóricas podem contribuir na fundamentação do PC como processo de pensamento humano?

- Quanto ao desenvolvimento do PC, quais os motivos da programação ser o principal conceito utilizado entre os conteúdos da CC?

Como estrutura, o trabalho contará com informações sobre definições e modelos do PC encontrados na literatura na seção 2, a relação entre programação e PC na seção 3 , seguindo para uma discussão sobre os conceitos basilares da CC e que podem servir como base para o desenvolvimento do PC, na seção 4 . A seção 5 tece apontamentos sobre uma nova possibilidade de investigar o PC, amparado nos pressupostos da THC e da TED. A seção 6 apontará as considerações finais. Por fim, são apresentadas as referências.

\section{Pensamento Computacional: definições e modelos}

O ponto de partida para a difusão do PC foi de Wing, que o descreveu como "uma habilidade fundamental para todos, não somente para cientistas da computação" e que "envolve a resolução de problemas, projeção de sistemas, e compreensão do comportamento humano, através da extração de conceitos fundamentais da ciência da computação" [Wing 2006]. Mais tarde, a autora, em colaboração com Jan Cuny e Larry Snyder, retomou a definição do PC, conceituando-o como "processos de pensamento envolvidos na formulação de problemas e suas soluções para que as soluções sejam representadas de uma forma que possam ser efetivamente realizadas por um agente de 
processamento de informações" [Cuny et al. 2010].

Em um trabalho colaborativo coordenado pela Computer Science Teachers Association (CSTA) e pela International Society for Technology in Education (ISTE), foi elaborada uma definição operacional para o PC, como "um processo de resolução de problemas" desde sua formulação, organização e análise dos dados, representação por meio de abstrações, como modelos e simulações, automatização das soluções utilizandose de ferramentas para resolvê-los da forma eficiente e eficaz, possibilitando a generalização deste processo para uma variedade de problemas [CSTA e ISTE 2011]. Outra organização científica, a Royal Society, também apresentou uma definição do PC, descrevendo-o como "o processo de reconhecer aspectos da computação no mundo que nos rodeia e de aplicar ferramentas e técnicas da Ciência da Computação para entender e raciocinar sobre sistemas e processos naturais e artificiais" [Royal Society 2012].

No Brasil, a Sociedade Brasileira de Computação (SBC), instituição que centraliza as discussões sobre o ensino de Computação no país, descreveu o PC como a "habilidade de compreender, definir, modelar, comparar, solucionar, automatizar e analisar problemas (e soluções) de forma metódica e sistemática" [SBC 2018]. O Centro de Inovação para a Educação Brasileira (CIEB), que desenvolveu um currículo de referência em tecnologia e computação para a Educação Infantil e Ensino Fundamental, também propôs uma definição para o PC, descrevendo-o como a "capacidade de sistematizar, representar, analisar e resolver problemas" [Raabe et al. 2018].

Outros pesquisadores prosseguiram analisando a temática e contribuindo com a discussão sobre sua definição. Tendo a resolução de problemas como elemento presente na descrição do PC, [Selby e Woollard 2013], pesquisaram os termos utilizados em sua conceituação e, com base nos mais empregados, definiram o PC como "uma abordagem focada na resolução de problemas, incorporando processos de pensamento que utilizam abstração, decomposição, design algorítmico, avaliação e generalizações". Na mesma direção, [Shute et al. 2017] o definiram como "a base conceitual necessária para resolver problemas de forma eficaz e eficiente (ou seja, algoritmicamente, com ou sem a ajuda de computadores) com soluções que são reutilizáveis em diferentes contextos".

Em uma recente pesquisa secundária, por meio da análise de definições do PC, [Fantinati e Santos Rosa 2021] descreveram o PC como um modo de pensar, originário da CC que, "quando aplicado por meio de estratégias, recursos e ferramentas computacionais ou não, pode contribuir para o desenvolvimento de habilidades, como o pensamento lógico, algorítmico e crítico, com vistas à resolução de situações-problema" seja em ambientes escolares ou na vida em sociedade.

Além da definição do termo PC, há trabalhos que têm apresentado propostas de desenvolvimento de taxonomias, frameworks ou modelos que compreendem os elementos ou componentes do PC. [Hsu et al. 2018] propuseram uma taxonomia para o PC baseada na definição e nos processos descritos por [Wing 2006], com o incremento de novos elementos, totalizando 19 processos do PC: abstração, projeto de algoritmo, decomposição, reconhecimento de padrões, coleta, representação e análise de dados, automação, paralelização, generalização, simulação, transformação, lógica condicional, conexão a outros campos, visualização, detecção e correção de erros, eficiência e desempenho, modelagem e solução de problemas.

Em uma Revisão Sistemática de Literatura, [Palts e Pedaste 2020] investigaram propostas de definição e modelos do PC em trabalhos desde Wing, em 2006 até o ano de 
2017. A análise de 65 trabalhos presentes no corpus documental considerou seis grupos de dimensões (elementos) do PC, identificando características frente à definição inicial de [Wing 2006]. Com base nessa análise, os autores elaboraram um novo modelo para o desenvolvimento do PC, que conta com três etapas compostas por 10 estágios: 1) Definição do problema (formulação, abstração, reformulação, decomposição); 2) Resolução do problema (coleta e análise de dados, projeto de algoritmos, paralelização e iteração, automação), e; 3) Análise da solução (generalização, testes e avaliação).

Apesar desse montante de informações sobre o PC, precisamos direcionar estudos para compreender como ele tem sido desenvolvido por meio dos conceitos computacionais, mais especificamente da $\mathrm{CC}$, visto que, diversos trabalhos têm apontado a programação como o principal conceito sob o qual o PC tem se desenvolvido [Tikva e Tambouris 2021, Zhang e Nouri 2019]. Assim, reforçando a segunda questão deste trabalho, verificaremos como a associação entre programação e PC tem sido estabelecida.

\section{Relação entre Pensamento Computacional e Programação}

Desde o trabalho de Papert, em 1980, a programação vem sendo considerada uma importante ferramenta no desenvolvimento do PC e, apesar de trabalhos descreverem que o PC não se resume a programação [Shute et al. 2017, Grover e Pea 2013], poucas são as pesquisas que consideram outros conceitos da Computação como componente principal no desenvolvimento do PC.

Em uma pesquisa secundária, [Tikva e Tambouris 2021] mapearam e analisaram 101 estudos e, por meio deles, desenvolveram um modelo conceitual de PC visando auxiliar na compreensão da temática e apoiar sua integração em práticas educacionais, tendo como conceito base da Computação, unicamente, a programação. Para eles, "a programação apoia o desenvolvimento do PC enquanto o PC fornece a programação de uma nova função atualizada". [Zhang e Nouri 2019] utilizam o Scratch como ferramenta para verificar quais as habilidades do PC podem ser obtidas no desenvolvimento do PC por meio de programação. Os conceitos computacionais abordados pelos trabalhos investigados se restringiram, portanto, a conceitos de programação tais como estruturas sequenciais, de repetição, condicionais, variáveis, lógica booleana, paralelismo e eventos.

Com outro viés, [Valente et al. 2017] realizaram uma análise referente à motivação econômica gerada pelas necessidades do mercado de trabalho por profissionais com conhecimento em PC. Segundo os autores, a premência por pessoas com tal capacitação acaba não permitindo uma formação de qualidade e o que deveria ser uma iniciativa de desenvolvimento do PC, acaba, de modo geral, reduzido ao ensino de uma linguagem de programação, ou seja, a formação de um programador para a indústria de desenvolvimento de software.

De maneira geral, temos vários trabalhos relacionando diretamente programação ao PC, seja como meio ou recurso para atingir determinado objetivo, por exemplo, como ferramenta na produção de jogos ou na manipulação de um robô, ou como fim, valendose de uma linguagem de programação em sua aprendizagem. Entre essas linguagens, as ferramentas de programação visuais, tal como o Scratch, têm sido amplamente utilizadas [Sousa et al. 2020, Araujo et al. 2020]. Em todos os casos, temos a programação diretamente relacionada ao PC, o que nos leva a refletir sobre a importância da programação como objeto de conhecimento dentro da Computação como ciência.

Apesar de alguns trabalhos destacarem que o PC tem sido desenvolvido em 
atividades da $\mathrm{CC}$, em geral, não fica explícito quais conceitos dessa área são abordados, excetuando-se a programação. O que nos leva a investigar os conceitos fundamentais da CC. Seria mesmo a programação o principal conceito dentro da área? Além dela, quais outros conteúdos podem ser objeto de estudo para o desenvolvimento do PC?

\section{Explorando a Ciência da Computação}

Em meados dos anos 2000, Wing descreveu que uma das motivações que a levaram a escrever seu trabalho sobre PC [Wing 2006], foi o fato de que a CC passava por um momento de apreensão, visto que as matrículas nos cursos de graduação estavam diminuindo [Wing 2014].

De fato, a autora conseguiu direcionar a atenção para a área, por meio de sua definição do PC. Não ficou esclarecido, porém, quais os conceitos da CC deveriam ser utilizados para promover o PC. E, apesar de descrever que "Ciência da computação não é programação" e que "pensar como um cientista da computação significa mais do que ser capaz de programar um computador" [Wing 2006], surgem algumas dúvidas: o que seria efetivamente pensar como um cientista da computação? Como não cientistas da computação podem desenvolver o PC sem conhecer os conceitos da CC? Mesmo com suas pistas descrevendo termos da área, como "deadlock", "backtracking", "Teste de Turing", entre outros, esses questionamentos permanecem sem resposta em sua obra.

Dessa maneira, investigamos quais os principais conceitos computacionais na área, mais especificamente no curso de graduação de CC, curso de formação para os cientistas da Computação. A obra "Ciência da Computação: uma visão abrangente", de [Brookshear 2013], oferece um cenário geral sobre a área, destacando as "ferramentas abstratas" ou componentes teóricos: Arquitetura de Máquina (Arquitetura de Computadores), Sistemas Operacionais, Redes de Computadores, Algoritmos, Linguagens de Programação, Engenharia de Software, Estruturas de Dados, Manipulação de Arquivos, Banco de Dados, Inteligência Artificial e Teoria da Computação.

De forma institucionalizada, a SBC vem conduzindo as discussões sobre os referenciais para os cursos de graduação em Computação no Brasil. Os currículos de referência propostos pela SBC serviram como base para a criação das Diretrizes Curriculares Nacionais (DCNs) para Cursos de Graduação em Computação. Os referenciais de formação para os cursos de graduação em computação mais atuais [ZORZO 2017] foram elaborados com base em três documentos: DCNs para os Cursos de Graduação em Computação [MEC 2016]; currículos de referência da SBC [SBC 2005], e; currículo da Association for Computing Machinery e Institute of Electrical and Electronic Engineers [ACM e IEEE 2013].

Os referenciais [ZORZO 2017] apresentam os conceitos fundamentais da Computação, apesar de terem sido elaborados com base na noção de competência. Esses conceitos, quando comparados às áreas de conhecimento do currículo da [ACM e IEEE 2013] e das matérias base do currículo de referência [SBC 2005], apresentam algumas diferenças, mas o cerne dos conceitos computacionais pode ser destacado nos três.

Assim, com base nos temas convergentes desses referenciais, destacamos os conceitos fundamentais na formação em CC: Algoritmos e Estrutura de Dados, Análise de Algoritmos, Arquitetura e Organização de Computadores, Banco de Dados, Computação Gráfica, Computação Paralela e Distribuída, Engenharia de Software, Inteligência Artificial, Interação Humano-Computador, Linguagens de Programação, 
Redes de Computadores, Segurança de Sistemas Computacionais, Sistemas Operacionais, Teoria da Computação e Teoria dos Grafos. Ressaltamos que esses conceitos foram elencados com base apenas na área computacional, ou seja, mesmo a CC tendo suas origens na Matemática, disciplinas dessa área não foram consideradas, nem mesmo das áreas da Física, Eletrônica e Contexto Social e Profissional [SBC 2005].

Desse modo, incrementamos os questionamentos que findaram a seção predecessora, com a seguinte indagação: considerando a área da Computação sendo formada por, pelo menos, 15 conceitos fundamentais ou subáreas, quais os motivos da programação ser o conceito preponderante nas pesquisas sobre o desenvolvimento do PC?

Visando fornecer subsídios para responder os questionamentos apresentados, uma nova fundamentação teórica para o PC se faz necessária.

\section{Nova perspectiva teórica}

No cenário estabelecido, avistamos inúmeras propostas de definição para o $\mathrm{PC}$, de modelos, que ampliam as possibilidades para as pesquisas sobre o PC. De maneira geral, temos estudos relacionando o ensino da programação para promover o desenvolvimento do PC por meio de intervenções educacionais, que avaliam as habilidades adquiridas pelos alunos ao realizar determinadas atividades. E, apesar de trabalhos como [DíazLauzurica e Moreno-Salinas 2019] indicarem contribuições dos fundamentos construtivistas de Jean Piaget, construcionismo de Seymour Papert e da teoria de Vygotsky, pouco tem sido o aprofundamento teórico no que tange aos processos de ensino, de aprendizagem e desenvolvimento humano.

Além de um aporte teórico adequado, [Kaminski et al. 2021] destacaram que é fundamental a organização das práticas pedagógicas, voltadas ao movimento de integrar o PC na escola. Para os autores, a inserção do PC na Educação Básica ainda necessita de investigações sobre "fundamentações teóricas, em termos didáticos e pedagógicos" [Kaminski et al. 2021], ou seja, devemos analisar tanto os conteúdos curriculares abordados, bem como avançar na compreensão das bases teórico-metodológicas nas quais os trabalhos estão alicerçados.

Por essa razão, acreditamos que pesquisas amparadas nos preceitos da THC e na TED, podem trazer contribuições significativas para o aprofundamento da compreensão do desenvolvimento do PC como um processo de pensamento, assim como tem contribuído para o entendimento da especificidade de tipos de pensamento como o filosófico, crítico, teórico, entre outros.

Especificamente em pesquisas sobre o pensamento teórico, enxergamos aproximações com o PC. Tendo em vista que o processo de formação ou desenvolvimento do pensamento teórico tem sido objeto de estudo de muitas pesquisas há tempo considerável, vislumbramos possibilidades de avançar na compreensão do $\mathrm{PC}$, bem como nos meios para o seu desenvolvimento, aproximando-nos de investigações acerca da formação do pensamento teórico. Esse tipo de pensamento é formado pela educação formal, por meio da aprendizagem de conceitos sistematizados pelas diferentes ciências, como afirma [Sforni 2019] a "aprendizagem de conceitos científicos, propiciada pela educação escolar, tem papel decisivo na formação dos sujeitos, possibilitando a tomada de consciência e o desenvolvimento do pensamento teórico". Trata-se de um pensamento não vinculado a uma futura atuação profissional específica, mas à formação integral dos seres humanos, da qual deve se ocupar a educação básica. 
Para [Vygotsky 2008], os conceitos científicos possuem um "sistema hierárquico de inter-relações", um sistema de conceitos que não são absorvidos completamente formados, tendo a instrução e a aprendizagem um papel fundamental na sua aquisição. Ao encontro dessa ideia, [Sforni 2015] argumenta que o conceito deve estar contido nas tarefas de aprendizagem para ser apropriado como instrumento do pensamento. Ou seja, todo processo de pensamento deve estar amparado em conceitos científicos e, sendo o PC um desses processos, consideramos que deve estar fundamentado nos conceitos base da CC. A própria [Wing 2006] descreveu que o PC "compartilha com o pensamento científico as maneiras gerais pelas quais podemos abordar a compreensão da computabilidade, da inteligência, da mente e do comportamento humano".

Para [Libâneo 2004], o pensamento teórico vai sendo formado ao mesmo tempo em que ações mentais vão sendo desenvolvidas por meio da solução de problemas e, desse modo, o estudante "assimila o conhecimento teórico e as capacidades e habilidades relacionadas a esse conhecimento". O autor ainda destaca que a formação do pensamento teórico ocorre pelo "domínio dos procedimentos lógicos do pensamento que, pelo seu caráter generalizador, permite sua aplicação em vários âmbitos da aprendizagem”.

Mesmo em estudos sobre o pensamento teórico, podemos observar aproximações com o PC, por exemplo, quando são mencionados os termos "solução de problemas" e "generalização". Em uma análise sobre a resolução de problemas, [Zankov 1984], contemporâneo de Vygotsky, há mais de 30 anos, mesmo sem qualquer informação sobre PC ou suas características, poderia estar inaugurando uma proposta de definição, ao afirmar que na solução de um problema deve-se "ter uma ideia clara do problema, de suas partes e que informação cada uma delas fornece. Deve saber encontrar o enunciado e a pergunta do problema de qualquer forma que apareçam no texto".

Com o exposto, propomos uma alternativa para ampliar a discussão sobre as pesquisas relacionadas ao PC. Estamos interessados em compreender a formação do pensamento humano, estendendo a investigação a outras formas de pensamento, especificamente o teórico e o computacional, analisando suas relações com os processos de ensino, aprendizagem e desenvolvimento humano. Esta nova perspectiva teórica tem como bases conceituais, além dos conceitos científicos da CC, os fundamentos da THC e da TED, particularmente, as proposições de [Davidov 1988].

Com essa orientação teórica, identificados e mapeados os conceitos fundamentais da $\mathrm{CC}$, será proposta uma estrutura conceitual do PC para que, amparados por estudos dos campos da Psicologia da Educação e Didática, possamos realizar uma organização do ensino e conduzir experimentos didáticos investigando o potencial dessa forma de organizar o ensino na aprendizagem e no desenvolvimento dos alunos. Os princípios didáticos e as ações docentes concebidas por [Sforni 2015] serão direcionadores para a realização dos procedimentos didáticos. Desse modo, almejamos colaborar com o arcabouço teórico e conceitual sobre o PC, além de apontar possibilidades para a organização do ensino, amparados em orientações teóricas diversas do que tem sido encontrado na literatura.

\section{Considerações Finais}

Apesar de recente, a temática do PC apresenta numerosos estudos e pesquisas que têm buscado definir e criar estruturas conceituais, propor formas para seu desenvolvimento, além de avaliá-lo, seja em trabalhos com caráter teórico ou por meio de iniciativas que 
promovem intervenções educacionais, de modo experimental. De maneira geral, as propostas para o desenvolvimento do PC valem-se da programação, seja por meio de ferramentas visuais ou mesmo linguagens de programação tradicionais, na criação de jogos ou uso de robótica, para alcançar esse objetivo. Não há unanimidade sobre abordagens ou metodologias de ensino e de aprendizagem utilizadas para tal, visto que, cada pesquisa apresenta sua forma de abordar, desenvolver e avaliar o PC.

Essas constatações nos levaram a investigar dois questionamentos que se originaram com base nos trabalhos da área. $\mathrm{O}$ primeiro, quanto as possibilidades de bases teóricas que podem contribuir na fundamentação do PC como processo de pensamento humano, além da CC, observamos aproximações com a formação do pensamento teórico, propiciado pela apropriação dos conceitos científicos, que tem como base conceitual a THC. Compreendemos que tal apropriação ocorre por meio de uma organização do ensino que conduz o estudante durante a aprendizagem e que precede e acompanha seu desenvolvimento. Essa organização é objeto de estudo da TED e, dessa maneira, investigar seus fundamentos nos parece fundamental. Duas áreas que permeiam essas teorias são a Psicologia da Educação e a Didática e por isso, serão a base para o desenvolvimento dos procedimentos didáticos a serem contemplados no experimento didático sobre as ações de ensino para a formação do PC.

Em relação a segunda questão levantada, sobre considerarmos a programação como conceito da $\mathrm{CC}$ preponderante no desenvolvimento do $\mathrm{PC}$, realizamos um mapeamento dos conteúdos basilares da área, destacando ao menos, 15 subáreas essenciais e que podem servir como alicerce para o estabelecimento de uma estrutura conceitual para o PC. Nessas subáreas, investigaremos seus conceitos-chave que orientarão a organização do ensino e as ações didáticas para o desenvolvimento do PC. Destacamos que, assim como apontado por grande parte da literatura, concordamos que a programação é fundamento para a $\mathrm{CC}$, pois todos os modelos computacionais são construídos por meio dela, porém, como não consideramos o PC um conceito estritamente relacionado a sistemas computacionais, mas sim, como um processo do pensamento humano, não temos a programação como único e principal conteúdo da $\mathrm{CC}$.

Com essas proposições, almejamos colaborar com essa temática de estudo que tem sido amplamente pesquisada, mas que permanece com possibilidades a serem exploradas. Ao propor uma organização e procedimentos didáticos aproximando áreas de origens distintas, como a CC, a THC e a Didática, ampliamos ainda mais o universo de pesquisa sobre desenvolvimento do PC.

\section{Referências}

ACM \& IEEE (2013). Computer Science Curricula 2013: Curriculum Guidelines for Undergraduate Degree Programs in Computer Science. Final Report. New York, NY, USA. Disponível em: dx.doi.org/10.1145/2534860. Acesso em: 19 de jun. 2021.

Araujo, N., Primo, T., \& Pernas, A. (2020). OntoScratch: ontologias para a avaliação do ensino de Pensamento Computacional através do Scratch. In Anais do XXXI Simpósio Brasileiro de Informática na Educação, (pp. 1823-1832). Porto Alegre: SBC.

Brookshear, J. G. (2013). Ciência da Computação: Uma Visão Abrangente. Bookman Editora.

CSTA \& ISTE (2011). Operational definition of computational thinking. Disponível 
em: https://www.iste.org/explore/computational-thinking/computational-thinking-all. Acesso em: 19 jun. 2021.

Cuny, J., Snyder, L., \& Wing, J. M. (2010). Demystifying computational thinking for non-computer scientists. Unpublished manuscript, referenced in http://www.cs.cmu.edu/ CompThink/resources/TheLinkWing.pdf.

Davydov, V. V. (1988). Problems of Developmental Teaching. The Experience of Theoretical and Experimental Psychological Research. Excerpts (Part III). Soviet education, 30(10).

Denning, P. J. (2017). Remaining trouble spots with computational thinking. Communications of the ACM, 60(6), 33-39.

Díaz-Lauzurica, B., \& Moreno-Salinas, D. (2019). Computational thinking and robotics: A teaching experience in compulsory secondary education with students with high degree of apathy and demotivation. Sustainability, 11(18), 5109.

Fantinati, R. E., \& dos Santos Rosa, S. (2021). Pensamento Computacional: Habilidades, Estratégias e Desafios na Educação Básica. Informática na educação: teoria \& prática, 24(1 Jan/Abr).

Grover, S., \& Pea, R. (2013). Computational thinking in K-12: A review of the state of the field. Educational researcher, 42(1), 38-43.

Hsu, T. C., Chang, S. C., \& Hung, Y. T. (2018). How to learn and how to teach computational thinking: Suggestions based on a review of the literature. Computers \& Education, 126, 296-310.

Kaminski, M., Klüber, T., \& Boscarioli, C. (2021). Pensamento Computacional na Educação Básica: Reflexões a partir do Histórico da Informática na Educação Brasileira. Revista Brasileira de Informática na Educação, 29, 604.

Libâneo, J. C. (2004). A aprendizagem escolar e a formação de professores na perspectiva da psicologia histórico-cultural e da teoria da atividade. Educar em Revista, (24), 113-147.

MEC (2016). Diretrizes Curriculares Nacionais para os Cursos de Graduação em Computação. Resolução CNE/CES n ${ }^{\circ}$, de 16 de novembro de 2016. Disponível em: http://portal.mec.gov.br/docman/novembro-2016-pdf/52101-rces005-16-pdf/file. Acesso em: 19 de jun. 2021.

Papert, S. A. (1980). Mindstorms: Children, computers, and powerful ideas. Basic books.

Palts, T., \& Pedaste, M. (2020). A Model for Developing Computational Thinking Skills. Informatics in Education, 19(1), 113-128. doi:10.15388/infedu.2020.06

Raabe, A. L. A., Brackmann, C. P., \& Campos, F. R. (2018). Currículo de referência em tecnologia e computação: da educação infantil ao ensino fundamental. Centro de Inovação para a Educação Básica-CIEB.

Royal Society. (2012). Shut down or restart: The way forward for computing in UK schools. Disponível em: https://royalsociety.org/-/media/education/computing-inschools/2012-01-12-computing-in-schools.pdf. Acesso em: 19 jun. 2021.

SBC (2005). Currículo de Referência da SBC para cursos de Graduação em 
Bacharelado em Ciência da Computação e Engenharia da Computação. https://www.sbc.org.br/documentos-da-sbc/category/131-curriculos-de-referencia. Acesso em: 19 de jun. 2021.

SBC (2018). Diretrizes para ensino de Computação na Educação Básica. Disponível em: https://www.sbc.org.br/documentos-da-sbc/category/127-educacao. Acesso em: 19 de jun. 2021.

Selby C. e Woollard, J. (2013). Computational thinking: the developing definition. Disponível em: https://eprints.soton.ac.uk/356481/1/Selby_Woollard_bg_soton_ eprints.pdf. Acesso em: 19 jun. 2021.

Sforni, M. S. D. F. (2015). Interação entre didática e teoria histórico-cultural. Educação \& Realidade, 40(2), 375-397.

Sforni, M. S. D. F. (2019). Pesquisas sobre modos de organização do ensino: necessidades, metodologia e resultados. In: Sforni, M. S. de F.; Serconek, G. C.; Belieri, C. M. (org.) Aprendizagem conceitual e organização do ensino: experimentos didáticos na educação básica. 1. ed. Curitiba: Editora CRV, v. 1. p. 10-32.

Shute, V. J., Sun, C., \& Asbell-Clarke, J. (2017). Demystifying computational thinking. Educational Research Review, 22, 142-158.

Sousa, L., Farias, E., \& Carvalho, W. (2020). Programação em Blocos Aplicada no Ensino do Pensamento Computacional: Um Mapeamento Sistemático. In Anais do XXXI Simpósio Brasileiro de Informática na Educação, (pp. 1513-1522). Porto Alegre: SBC.

Tikva, C., \& Tambouris, E. (2021). Mapping computational thinking through programming in K-12 education: A conceptual model based on a systematic literature Review. Computers \& Education, 162, 104083.

Valente, J. A., Freire, F. M. P., Arantes, F. L., Amiel, T., \& Baranauskas, M. C. C. (2017). Alan Turing tinha Pensamento Computacional? Reflexões sobre um campo em construção. Tecnologias, Sociedade e Conhecimento, 4(1), 7-22.

Vygotsky, L. S. (2008). O desenvolvimento dos conceitos científicos na infância. Pensamento e linguagem. São Paulo: Martins Fontes, 103-148.

Wing, J. M. (2006). Computational thinking. Communications of the ACM, 49(3) 33-35.

Wing, J. M. (2008). Computational thinking and thinking about computing. Philosophical Transactions of the Royal Society A: Mathematical, Physical and Engineering Sciences, 366(1881), 3717-3725.

Wing, J. M. (2014). Computational thinking benefits society. 40th Anniversary Blog of Social Issues in Computing, 2014, 26.

Zankov, L. V. (1984). La enseñanza y el desarrollo. Traducción del ruso por Vicente Pertegaz. Moscú: Editorial Progreso, 1975. (Traducción al español, 1984).

Zhang, L., \& Nouri, J. (2019). A systematic review of learning computational thinking through Scratch in K-9. Computers \& Education, 141, 103607.

Zorzo, A. F., Nunes, D., Matos, E., Steinmacher, I., de Araujo, R. M., Correia, R., \& Martins, S. (2017). Referenciais de Formação para os Cursos de Graduação em Computação. Sociedade Brasileira de Computação (SBC). 153p, 2017. 